\title{
Some bifurcation diagrams for Taylor vortex flows
}

Rita Meyer-Spasche

Max Planck Institut für Plasmaphysik, D8046 Garching, West Germany

H. B. Keller

Applied Mathematics, California Institute of Technology 217-50, Pasadena, California 91125

(Received 3 May 1984; accepted 13 February 1985)

The numerical continuation and bifurcation methods of Keller [H. B. Keller, in Applications of Bifurcation Theory (Academic, New York, 1977), pp. 359-384] are used to study the variation of some branches of axisymmetric Taylor vortex flow as the wavelength in the axial direction changes. Closed "loops" of solutions and secondary bifurcations are determined. Variations with respect to Reynolds number show the same phenomena. The results presented here show that Taylor vortices with periodic boundary conditions exist in a wider range of wavelengths, $\lambda$, than observed in the Burkhalter/Koschmieder experiments [Phys. Fluids 17, 1929 (1974)]. They also show that there is possibly a $\lambda$ subinterval within the neutral curve of Couette flow such that there are no Taylor vortex flows with smallest period in this interval.

\section{INTRODUCTION}

We present numerical studies of steady axisymmetric Taylor vortex flows between two concentric, infinitely long cylinders. The outer one, at $\hat{r}=R_{2}$, is at rest and the inner one, at $\hat{r}=R_{1}$, is rotating with angular speed $\omega_{1}$. The Navier-Stokes equations used to compute these flows are, in dimensionless form:

$$
\begin{aligned}
& u_{r}+(1 / r) u+w_{z}=0, \\
& \Delta u-\left(1 / r^{2}\right) u-p_{r}=\operatorname{Re}\left[u u_{r}+w u_{z}-(1 / r) v^{2}\right], \\
& \Delta v-\left(1 / r^{2}\right) v=\operatorname{Re}\left[u v_{r}+w v_{z}+(1 / r) u v\right], \\
& \Delta w-p_{z}=\operatorname{Re}\left(u w_{r}+w w_{z}\right) .
\end{aligned}
$$

Here lengths have been scaled by $R_{1}$, velocities by $\omega_{1} R_{1}$,

$$
\Delta \equiv \frac{\partial^{2}}{\partial r^{2}}+\frac{1}{r} \frac{\partial}{\partial r}+\frac{\partial^{2}}{\partial z^{2}}
$$

and the Reynolds number is

$$
\mathrm{Re} \equiv \omega_{1} R_{1}^{2} / v,
$$

where $v$ is the kinematic viscosity of the fluid. The dimensionless gap width, $\delta \equiv\left(R_{2}-R_{1}\right) / R_{1}$ and radii ratio, $\eta \equiv R_{1} /$ $R_{2}$ are related by $\delta=\eta^{-1}-1$. A final pair of dimensionless parameters is introduced to denote the wavelength $\lambda$, or wavenumber $k$, of the flow in the axial direction. These parameters are related by $\lambda \delta \equiv 2 \pi / k$ and enter into the boundary conditions which require periodicity of period $2 \pi / k$. Specifically, the boundary conditions are

$$
\begin{aligned}
& u(1, z)=u(1+\delta, z)=0, \\
& w(1, z)=w(1+\delta, z)=0, \quad|z| \leqslant \pi / k, \\
& v(1, z)=1, v(1+\delta, z)=0, \\
& u(r,-\pi / k)=u(r, \pi / k), \\
& v(r,-\pi / k)=v(r, \pi / k), \quad 1 \leqslant r \leqslant 1+\delta . \\
& w(r,-\pi / k)=w(r, \pi / k)=0 .
\end{aligned}
$$

The problem posed by (1) and (2) is solved numerically by using Fourier expansions in $z$ and centered finite differences in $r$. The resulting system of nonlinear algebraic equations can be denoted by

$$
\mathbf{G}(\mathbf{x}, \boldsymbol{\alpha})=0
$$

where $x$ represents the values of the Fourier coefficients at the radial net points and $\alpha \equiv(\operatorname{Re}, \eta, k)$ represents the three independent parameter values. We also use other parameter combinations such as $\alpha \equiv(\operatorname{Re}, \eta, \lambda)$.

The indicated formulation of (3) is described in some detail in Ref. 1. These equations were solved, as reported there, with $\eta=0.95, \eta=0.5$, and $\lambda \approx 2$ using the pseudoarclength continuation and bifurcation methods of Ref. 2 with $\operatorname{Re}$ as the (variable) continuation parameter. Bifurcation from Couette flow was obtained in both cases and the development of the Taylor vortices up to $\operatorname{Re}=4.2 \mathrm{Re}_{\mathrm{cr}}$ was studied for $\eta=0.5$. These methods and simple continuation in $\lambda$ were also used in Ref. 3 to study variations with respect to $\lambda$ and $\operatorname{Re}$ for fixed $\eta=0.5$. Transitions were found between solutions with different numbers of vortices, and limit points or "folds" in the solution paths were detected. Previously, folds in Taylor vortex flows had only been observed for very short cylinders where the end effects are important. ${ }^{4}$ Spatial period halving bifurcations had been found in the study of steady gravity-capillary waves on deep water. $^{5}$

In the present work we study in more detail the structure of the families of solutions or flows with respect to continuous variation of both parameters $\lambda$ and Re. Indeed, all the continuation methods of Ref. 2 have been implemented for $\lambda$ as well as for $\operatorname{Re}$ and thus folds with respect to both parameters are easily detected and circumvented. New bifurcations have been determined and a "closed" bifurcation diagram has been obtained for $\operatorname{Re}=\sqrt{1.5} \operatorname{Re}_{\mathrm{cr}}, \eta=0.727$, and $\lambda$ varying between the bounds given by the neutral curve of simple circumferential Couette flow. These parameter values allow us to compare our results with the experiments in Ref. 6. Over this $\lambda$ interval we find up to six different steady axisymmetric flows with period $\lambda$. We find it useful to present the bifurcation diagram in terms of a quantity different from the torque, i.e., the radial velocity at the midpoint of the $r-z$ period rectangle.

In addition, another bifurcation diagram is obtained by 
varying $\operatorname{Re}$ with $\eta=0.727, \lambda=2.65$, and $\lambda=1.325$. This diagram again shows a closed path of solutions (an isola) and the spatial period doubling (or halving) bifurcation as $R e$ is varied. A similar series of computations for $\lambda=2$ and $\lambda=1$ does not show interactions between these branches.

In Sec. II we discuss some of the computational techniques and the "aliasing" properties inherent in representing periodic solutions. In Sec. III we discuss the specific Taylor vortex flows that were computed. The computations have been done on the California Institute of Technology campus machine, an IBM 370/3032.

\section{COMPUTATIONAL TECHNIQUES AND ALIASING}

The basic numerical methods we use have been discussed in detail in Refs. 1 and 2. We examine here some previously neglected details and some new techniques.

\section{A. Allasing and periodic solutions}

The periodic boundary conditions (2b) invite Fourier series representations of the solution. Thus we seek the radial velocity $u(r, z)$ in the form:

$$
u(r, z ; \mathfrak{a}, k) \equiv \sum_{j=1}^{N} a_{j}(r) \cos j k z .
$$

Here a denotes the Fourier coefficients used in the representation and $k$ is the wavenumber imposed in the formulation. Note, however, that for any integer $m$ we can also write this velocity as

$$
u\left(r, z ; \mathbf{b}, \frac{k}{m}\right) \equiv \sum_{j=1}^{m N} b_{j}(r) \cos j \frac{k}{m} z,
$$

provided

$$
b_{j}(r) \equiv\left\{\begin{array}{lll}
0 & \text { if } & j \neq 0(\bmod m) \\
a_{j / m}(r) & \text { if } & j \equiv 0(\bmod m) .
\end{array}\right.
$$

The wavenumbers used in these representations are different but they yield identical velocities. Thus it is possible for calculations with different wavenumbers to yield identical flows. Of course, more harmonics must be allowed for the smaller wavenumbers in order to obtain the same accuracy. We call these dual representations "aliasing representations." They can also be thought of as viewing the flow through two different windows, one $m$-times longer than the other.

Another change in representation is obtained by translating the $z$ axis half a period. Then $k z$ is replaced by $k z+\pi \equiv k \hat{z}$ and in the new $\hat{z}$ coordinates (4) becomes

$$
u(r, \hat{z} ; \mathbf{c}, k)=\sum_{j=1}^{N} c_{j}(r) \cos j k \hat{z},
$$

where

$$
c_{j}(r)=(-1) a_{j}(r) \text {. }
$$

Clearly, $u(r, z ; \mathbf{a}, k)$ and $u(r, z ; \mathbf{c}, k)$ represent different solutions in the same $z$ coordinates, but one can be obtained from the other by the indicated shift. Indeed, the familiar bifurcation from Couette flow yields both flows.

\section{B. Continuation in $\lambda$}

Using the parameters $\alpha \equiv(\operatorname{Re}, \eta, \lambda)$ in $(3)$ we assume that a solution $x_{0}$ is known for $\alpha=\alpha_{0}$; that is

$$
\mathbf{G}\left(\mathbf{x}_{0} ; \mathbf{R e}_{0}, \eta_{0}, \lambda_{0}\right)=0 \text {. }
$$

With $\operatorname{Re}_{0}$ and $\eta_{0}$ fixed we seek solutions for $\lambda \neq \lambda_{0}$. If the Jacobian matrix $\mathbf{G}_{x}^{0} \equiv \partial \mathbf{G}\left(\mathbf{x}_{0} ; \mathbf{R e}_{0}, \eta_{0}, \lambda_{0}\right) / \partial \mathbf{x}$ is nonsingular, then the implicit function theorem assures us that we can solve (3) for $\mathbf{x}(\lambda)$, with $\alpha=\left(\operatorname{Re}_{0}, \eta_{0}, \lambda\right)$ provided $\left|\lambda-\lambda_{0}\right|$ is sufficiently small. This is the basis for simple continuation:

$$
\begin{aligned}
& \text { (i) Pick } \lambda_{1} \text { with }\left|\lambda_{1}-\lambda_{0}\right| \text { small, set } \alpha=\left(\operatorname{Re}_{0}, \eta_{0}, \lambda_{1}\right) \text {; } \\
& \text { (ii) Set } \mathbf{x}^{0}\left(\lambda_{1}\right)=\mathbf{x}_{0} ; \\
& \text { (iii) Solve } \mathbf{G}_{\mathbf{x}}\left[\mathbf{x}^{\nu}\left(\lambda_{1}\right), \mathbf{\alpha}\right] \Delta \mathbf{x}^{v}=-\mathbf{G}\left[\mathbf{x}^{\nu}\left(\lambda_{1}\right), \mathbf{\alpha}\right] \text {; } \\
& \text { (iv) Set } x^{\nu+1}\left(\lambda_{1}\right)=\mathbf{x}^{\nu}\left(\lambda_{1}\right)+\Delta \mathbf{x}^{\nu} \text {; }
\end{aligned}
$$

(v) Test for convergence; return to (iii) if not terminated. This is Newton's method, and the step size $\left|\lambda_{1}-\lambda_{0}\right|$ can be adjusted as the iterations converge more or less rapidly. This procedure was used in Ref. 3.

As the path or branch of solutions parametrized by $\lambda$ approaches a limit point or a bifurcation point, the above procedure fails. We then employ a form of pseudoarclength continuation devised in Ref. 2. Basically we give up the representation $\mathbf{x}=\mathbf{x}(\lambda)$ and seek instead $\mathbf{x}=\mathbf{x}(\sigma)$ with $\lambda=\lambda(\sigma)$ to be determined. The new parameter $\sigma$, an approximation to arclength, is specified at each step. Having introduced a new parameter, we are free to impose one additional (scalar) constraint. This can be done in many ways but we have used here, essentially,

$$
\begin{aligned}
N(\mathbf{x}, \lambda ; \sigma) \equiv & \dot{\mathbf{x}}\left(\sigma_{0}\right)^{T} \cdot\left[\mathbf{x}(\sigma)-\mathbf{x}\left(\sigma_{0}\right)\right] \\
& +\dot{\lambda}\left(\sigma_{0}\right)\left[\lambda(\sigma)-\lambda\left(\sigma_{0}\right)\right]-\left(\sigma-\sigma_{0}\right)=0 .
\end{aligned}
$$

Now $\sigma$ takes the place of $\lambda$ in the above algorithm (7) but we solve (3) and (8) simultaneously for $[\mathbf{x}(\sigma), \lambda(\sigma)]$. In place of Jacobian $\mathbf{G}_{\mathbf{x}}$ in (iii) of (7) we have

$$
\left(\begin{array}{cc}
\mathbf{G}_{\mathbf{x}} & \mathbf{G}_{\lambda} \\
N_{\mathbf{x}} & N_{\lambda}
\end{array}\right) \equiv\left(\begin{array}{cc}
\mathbf{G}_{\mathbf{x}} & \mathbf{G}_{\lambda} \\
\dot{\mathbf{x}}\left(\sigma_{0}\right)^{T} & \dot{\lambda}\left(\sigma_{0}\right)
\end{array}\right) .
$$

It is shown in Ref. 2 that this matrix is nonsingular at simple folds while $\mathbf{G}_{\mathbf{x}}$ is singular; this accounts for the main effectiveness of this continuation method. Small steps in $\sigma$ are assured to converge. This same procedure has been used in Ref. 1 with $\lambda$ replaced by $\operatorname{Re}$. In the current application we use difference quotients to approximate $\mathbf{G}_{\lambda}$ rather than evaluate the exact derivative; quadratic convergence was still maintained.

\section{Numerical accuracy}

Computations with radial mesh widths $h=1 / 8, h=1$ / $16, h=1 / 32$ have been carried out and only quantitative but no qualitative changes were observed. Compare Fig. 1 in Refs. 1 and 3. Both figures show very clearly that the theoretically expected $\mathscr{O}\left(h^{2}\right)$ error dependence is obtained and that the value $h=1 / 32$ used throughout the current numerical work yields good approximations for the moderate range of Reynolds numbers covered here.

The errors caused by truncating the Fourier expansions 


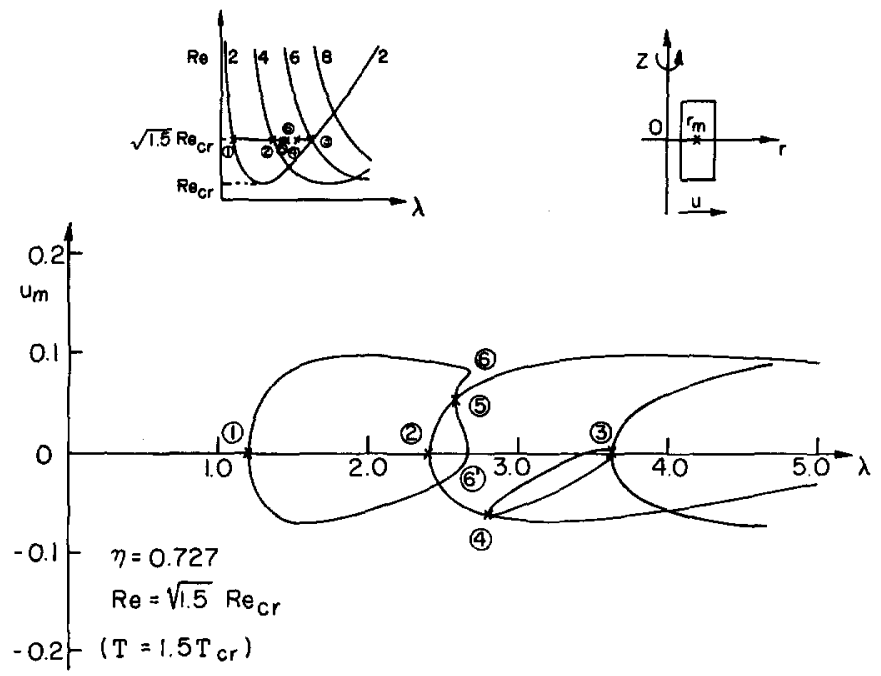

FIG. 1. Variation with the period $\lambda$ of the radial velocity at the "midpoint" $u_{m}$. Couette flow has $u \equiv 0$ and from it bifurcations occur at (1) and (3) of two-vortex flows, at (2) of four-vortex flow. At (4) and (5) period doubling bifurcations between branches of two- and four-vortex flows occur. Folds in branches of two-vortex flows occur at (6) and (6).

are of a different nature and easily cause qualitative changes-solutions can disappear. Naturally this effect is related to the number of harmonics used for a given value of $\lambda$. For example, one harmonic is sufficient to approximate the two vortex solutions that bifurcate from Couette flow for $\operatorname{Re}$ just above $\operatorname{Re}_{\mathrm{cr}}$ with $\lambda=2$. When $\lambda=4$ is used we need two harmonics to get the same solution. In all of the current computations $N=6$ harmonics are employed. This yields sufficient accuracy for $\lambda \leqslant 4$ and it allows us to compute the basic Taylor vortices up to $\lambda=12$.

\section{COMPUTED TAYLOR VORTEX FLOWS}

It is difficult to display the rich variety of solutions and their relations to each other in simple plane graphs. We have chosen to do this by plotting the radial velocity at the center of our computational domain, $u_{m} \equiv u(1+\delta / 2,0)$ vs $\lambda$ and vs Re. Positive $u_{m}$ corresponds to outward directed flow. We also present a graph of "poloidal kinetic" energy versus $\lambda$. This is similar to the torque graphs usually given in studies of Taylor vortex flows. But as we shall see, the velocity graphs contain much more information.

\section{A. The $\lambda$-continuation paths}

Our main results are summarized in Fig. 1 where $u_{m}$ vs $\lambda$ is plotted for fixed $\eta=0.727$ and $\operatorname{Re}=\sqrt{1.5} \operatorname{Re}_{\mathrm{cr}}$. We first describe how the numerical continuation and bifurcation techniques were used to obtain these results.

Starting with Couette flow at $\eta=0.727, \lambda=2$, and $\mathbf{R e}_{\mathrm{cr}} \doteq 219.10$ (the determinant of the Jacobian matrix $\mathbf{G}_{\mathbf{x}}$ changes sign at $\operatorname{Re}_{\mathrm{cr}}$ ), one of the branch switching techniques of Ref. 2, as described in Ref. 1, was used to compute the Taylor vortex flows bifurcating from Couette flow. This bifurcating branch of solutions was then continued in $\mathbf{R e}$, using pseudoarclength continuation, up to $\operatorname{Re} \doteq \sqrt{1.5} \mathbf{R e}_{\mathrm{cr}}$. Actually only half of the branch was computed since symmetry, via the shift in $z$ coordinate by $\pi / k$, yields the other half branch. (Recall that the bifurcation from Couette flow is of the "supercritical" or "pitchfork" type.)

Starting from one of the two bifurcated Taylor vortex flows at $\eta=0.727, \operatorname{Re}=\sqrt{1.5} \operatorname{Re}_{\mathrm{cr}}, \lambda=2$ we then do simple continuation in $\lambda$, both up and down, using the values $\lambda=2(0.05) 2.65$ and $\lambda=2(-0.05) 1.2$. The decreasing $\lambda$ continuation switches back to Couette flow at $\lambda=1.2$ since there is apparently no other neighboring solution. Further, the Taylor vortex flows go smoothly to the Couette flow along this decreasing $\lambda$ traverse. However, the increasing $\lambda$ continuation becomes more difficult and fails to converge at $\lambda=2.70$. Thus we switch to pseudoarclength continuation at $\lambda=2.65$, and this procedure automatically finds a limit point or fold at $\lambda \doteq 2.6754$. This corresponds to the point labeled (6) in Fig. 1. Before this fold is reached, two additional vortices have formed where the flow toward the inner cylinder has slowed down. As this path of solutions is traversed, the new weak vortices grow in magnitude and the flow changes continuously to a four-vortex flow. How this change develops is illustrated by plots of streamlines and level lines of $u, v, w$, and $p$ in Fig. 3 and 4 of Ref. 3. Though these plots were done for different parameter values $\left(\operatorname{Re}=\sqrt{1.3} \operatorname{Re}_{\mathrm{cr}}, \lambda\right.$ varying, $\left.\eta=0.5\right)$, the pictures for the case considered here are very similar. Even the streamlines displayed in Fig. 3 of Jones ${ }^{7}$ are very similar, though they deal with the case of counter-rotating cylinders ( $R e$ varying, $\lambda=2, \eta=0.5$ ).

Upon passing around the fold the continuation procedure decreases $\lambda$ and near $\lambda=2.59$, labeled (5) in Fig. 1, the solution joins smoothly to the other branch of the Taylor vortex flows which bifurcated from Couette flow at (1). We detect this joining up of the two branches by means of a simultaneous sign change in all of the odd Fourier mode coefficients at all of the mesh points. This is equivalent to a shift along the $z$ axis by the amount $\pi / k$. So in effect we have determined a closed loop or family of solutions, parametrized by $\lambda$, with fixed $\eta$ and Re. This loop contains the points (1) $\rightarrow$ (6) $\rightarrow$ (5) $\rightarrow$ (6) $\rightarrow$ (1) in Fig. 1. The plotted closed curve is not symmetric since $u_{m}=u(1+\delta / 2,0)$ is the ordinate. If we had used $-u_{m}(1+\delta / 2, \pm \pi / k)$ as the ordinate we would obtain the loop essentially rotated $180^{\circ}$ about the line joining (1) and (5). The point (5) turns out to be on the branch of four-vortex solutions that bifurcate from Couette flow at (2). Careful numerical tests ensure that this fourvortex branch is the periodic repetition of the two-vortex branch bifurcating at (1). Thus we have also found a secondary bifurcation between a two-vortex branch and a fourvortex branch. However, we stress that neither branch remains a pure two- or four-vortex flow; they are named for the flow developed when bifurcation from Couette flow occurs.

At the point labeled (3) a multiple bifurcation from Couette flow occurs; both two-vortex and six-vortex flows occur. The branch of two-vortex flows exists for decreasing $\lambda$ values, and as the period decreases two more vortices form. Now, however, they form where the flow is outward. Continued decrease of $\lambda$ yields strengthened vortices, and eventually this branch joins the same four-vortex branch that bifurcated from Couette flow in the above discussion. Thus another secondary bifurcation has been found as well as an- 


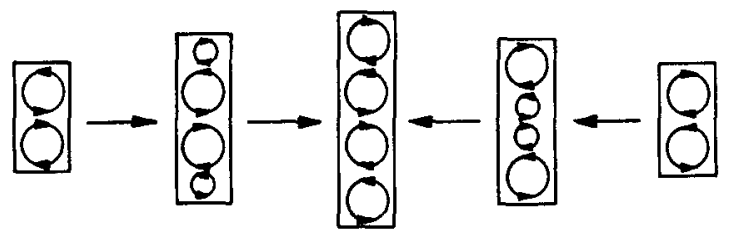

(1) UPPER BRANCH

(5) LOWER BRANCH

(1)

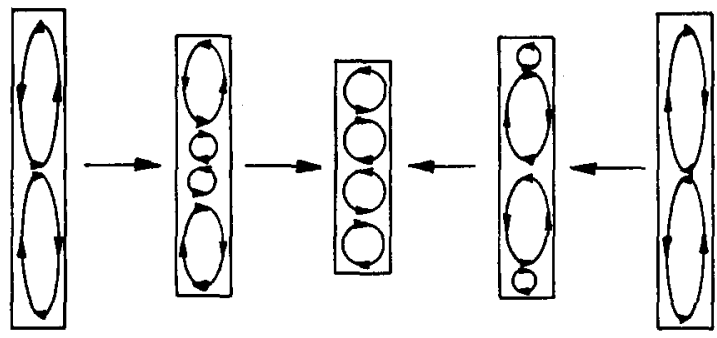

(3)

UPPER BRANCH

(4) LOWER BRANCH

(3)

FIG. 2. Schematic diagram of how the cellular structure of Taylor vortex flows change with wavelength between two-cell and four-cell flows. The sketches indicate the transitions with $\lambda$ along the upper and lower paths (1) $\rightarrow$ (5) and (3) $\rightarrow$ (4) of Fig. 1 .

other closed loop of solutions joining (3) and (4) (see Fig. 1). Streamlines of the changes that occur along such a solution branch can be seen in Fig. 5 of Ref. 3. In Fig. 2 we illustrate schematically both changes (1) - (6)- (5) and (3) - (4).

In Fig. 3 the branches of solutions described above are plotted in terms of their poloidal kinetic energy. A plot of torque versus $\lambda$ would be similar. The critical points (1),...,6) are indicated on the graph. Note how much more information on changes of solutions with $\lambda$ is contained in Fig. 1 than in Fig. 3.

The value $R e=\sqrt{1.5} \operatorname{Re}_{c r}$ was chosen for these investigations for two reasons. First, a multiple bifurcation occurs at this Re value. Second, the range of wavelength in the experiments of Burkhalter and Koschmieder is quite wide in a neighborhood of this Re value. They generated Taylor vortex flows with wavelength $\lambda=1.5$ by sudden starts (see Fig. 9 in Ref. 6). Taylor vortices with $\lambda=2.106$ and $\lambda=2.108$ were produced by filling experiments and by sudden starts, respectively. These were observed for Re numbers larger and smaller than, but close to $\sqrt{1.5} \operatorname{Re}_{\mathrm{cr}}$ (see Figs. 7 and 10 of Ref. 6). Figure 4 is an extension of Fig. 1, with more periodic repetitions. We have also included (rounded) numerical values of the critical wavelengths for easier comparison with the Burkhalter/Koschmieder experiments.

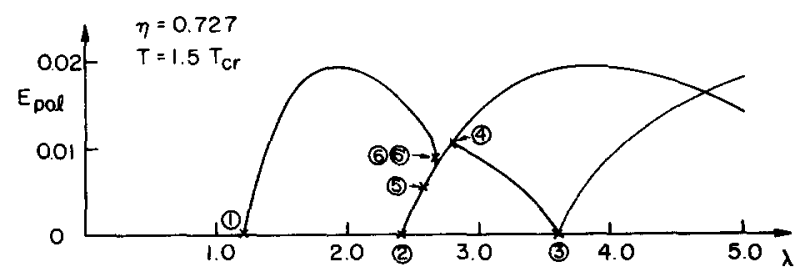

FIG. 3. Poloidal kinetic energy $E_{\text {pol }}$ versus period $\lambda$. The same branches and critical points as in Fig. I are shown. Distinct branches with the same $E_{\mathrm{pol}}$ coincide here and so important details are concealed. A torque versus $\lambda$ graph would be very similar.

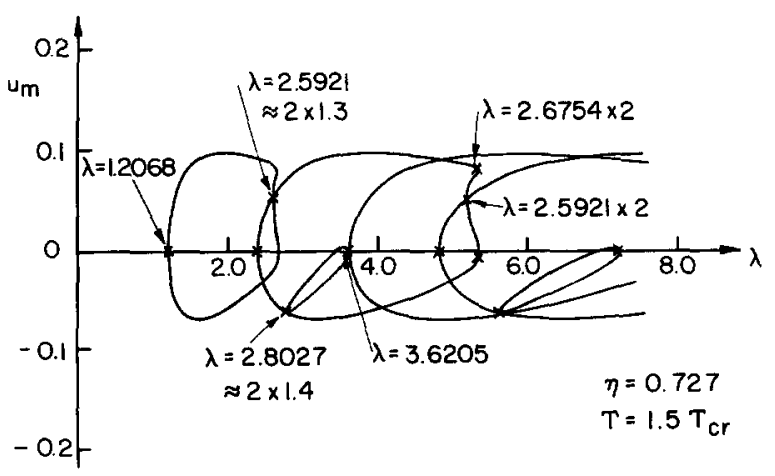

FIG. 4. As in Fig. 1 but higher period branches are also shown. The multiple bifurcation at $\lambda=\lambda * \equiv 3.6205$ contains Couette flow, two-vortex flows for $\lambda \leqslant \lambda *$ and six-vortex flows for $\lambda>\lambda *$. The experiments of Burkhalter and Koschmieder include the interval $1.5<\lambda<2.108$ for the indicated $\eta$ value and an interval of $T$ including $1.5 T_{\mathrm{cr}}$.

It has generally been assumed that the convex (parabolic shaped) neutral curves indicating bifurcations from Couette flow separate the $(\operatorname{Re}, \lambda)$ plane into regions where $2 n$ vortex solutions exist (above) and into regions where they do not exist (below) the $2 n$th "parabola" (see inset in upper portion of Fig. 1). But this assumption is incorrect. It is shown in Refs. 3 and 8 that for some fixed $\eta$ and $\lambda$, the bifurcation with respect to $\operatorname{Re}$ is subcritical. Thus for such $\eta$ and $\lambda$ values there exist solutions below the neutral curve. Our current calculations indicate that the converse may also be true. This is suggested by the "gap" in the $\lambda$ traverse shown in Fig. 1 (upper inset), where for the $\lambda$ interval between (6) and (4) no two-vortex branch solutions have been found.

\section{B. Re continuation paths}

For the period $\lambda=2.65$ we have done continuation with respect to $\mathrm{Re}$, or rather, $T \sim \mathrm{Re}^{2}$ (see Fig. 5). This $\lambda$ value is situated between the two folds at (6) and (6) and the secondary bifurcation at (5). As shown in Fig. 5 we find two folds with respect to $T$ on the $\lambda=2.65$ branch and a periodhalving bifurcation to a $\lambda=1.325$ branch. This figure is

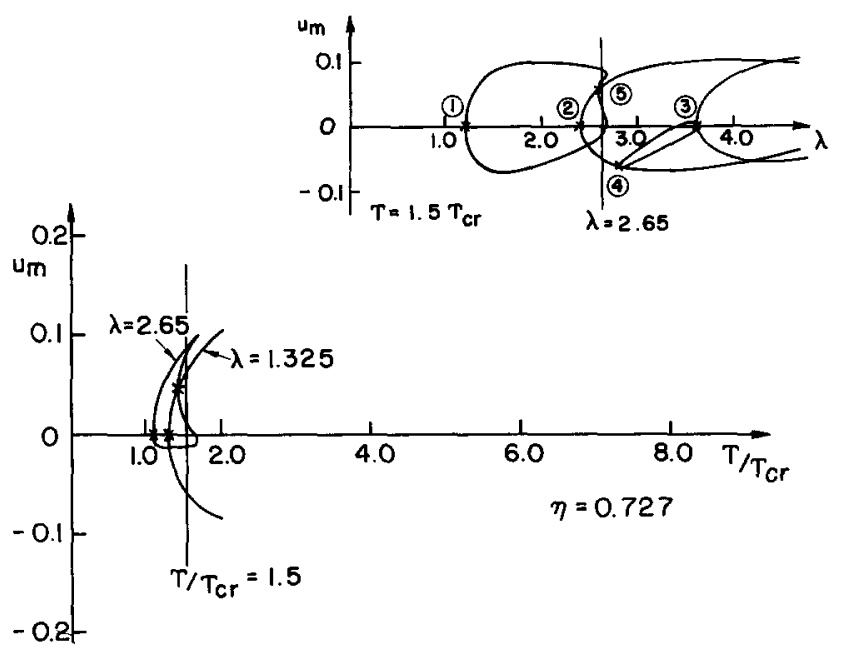

FIG. 5. Variation with the Taylor number, $T \equiv \mathbf{R e}^{2}$, of the radial velocity $u_{m}$. The solution branch for $\lambda=1.325$ has a period doubling bifurcation to the branch for $\lambda=2.65$. 


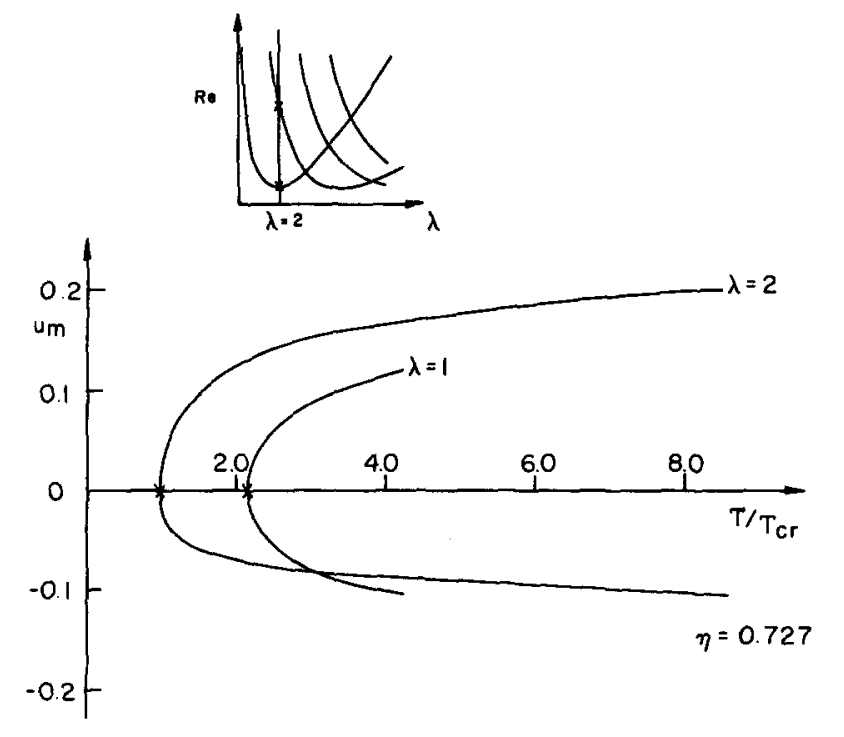

FIG. 6. As in Fig. 5, but solution branches for $\lambda=1$ and $\lambda=2$ are shown and they do not interact (i.e., no bifurcation between them for the indicated Re range).

drawn to the same scale as Fig. 6, in which $T$ continuation for the $\lambda=1$ and $\lambda=2$ branches is shown. No interaction of these branches has been found. This agrees with the computations of Jones, ${ }^{7}$ who used $\eta=0.8756$.

\section{ACKNOWLEDGMENTS}

We wish to thank F. Busse, D. Coles, D. Knight, P. Saffman, and the referees for useful comments about this work.

This work was supported by the Department of Energy under Contract No. DE-AT03-76ER 72012 and by the Army Research Office under Contract No. DAAG-29-81K-0107.

'R. Meyer-Spasche and H. B. Keller, J. Comp. Phys. 35, 100 (1980). ${ }^{2} \mathbf{H}$. B. Keller, in Applications of Birfurcation Theory, edited by P. Rabinowitz (Academic, New York, 1977), pp. 359-384.

${ }^{3} G$. Frank and R. Meyer-Spasche, Z. Angew. Math. Phys. 32, 710 (1981).

T. B. Benjamin, Proc. R. Soc. London Ser. A 359, 1, 27 (1978).

${ }^{5}$ B. Chen and P. Saffman, Stud. Appl. Math. 60, 183 (1979); 62, 95 (1980).

6J. E. Burkhalter and E. L. Koschmieder, Phys. Fluids 17, 1929 (1974).

${ }^{7}$ C. A. Jones, J. Fluid Mech. 120, 433 (1982).

${ }^{8}$ I. P. Andreichikov, Izv. Akad. Nauk SSSR, Mekh. Zhidk. Gaza, No. 2, 150 (1975). 